\title{
Minimal periods of maps of rational exterior spaces
}

\author{
by \\ Grzegorz Graff (Gdańsk)
}

\begin{abstract}
The problem of description of the set $\operatorname{Per}(f)$ of all minimal periods of a self-map $f: X \rightarrow X$ is studied. If $X$ is a rational exterior space (e.g. a compact Lie group) then there exists a description of the set of minimal periods analogous to that for a torus map given by Jiang and Llibre. Our approach is based on the Haibao formula for the Lefschetz number of a self-map of a rational exterior space.
\end{abstract}

1. Introduction. Let $f: X \rightarrow X$ be a self-map of a topological space $X$. For $m \geq 1$ we define $P^{m}(f)=\operatorname{Fix}\left(f^{m}\right)$ and $P_{m}(f)=P^{m}(f) \backslash$ $\bigcup_{0<n<m} P^{n}(f)$. The last is the set of $m$-periodic points of $f$. If $P_{m}(f) \neq \emptyset$ then $m$ is called a minimal period of $f$. The set of all minimal periods of $f$ is denoted by $\operatorname{Per}(f)$.

The classical Lefschetz theorem states that for a self-map $f$ of a nice space (e.g. finite CW-complex, compact manifold) if $L(f) \neq 0$ then $\operatorname{Fix}(f) \neq \emptyset$. Applying this theorem to the $m$ th iteration $f^{m}$ we find that $L\left(f^{m}\right) \neq 0$ implies $P^{m}(f) \neq \emptyset$, but there is no information about $P_{m}(f)$. Another classical fixed point theorem, the Lefschetz-Hopf formula, says that $L\left(f^{m}\right)=$ $I\left(f^{m}, X\right)$, where $I\left(f^{m}, X\right)$ is the fixed point index of $f^{m}$. Again a direct application of this relation to the iterations of $f$ does not pick up minimal periods in general.

Note that the Lefschetz number is defined as the alternating sum of the traces of the maps induced by $f$ on the cohomology spaces of $X$. This yields some properties of the sequence $\left\{L\left(f^{m}\right)\right\}_{m=1}^{\infty}$ and consequently $\left\{I\left(f^{m}, X\right)\right\}_{n=1}^{\infty}$ such as fulfilment of congruences (called Dold's relations), rationality of the generated zeta function, and others (cf. [D], [BB], [MP]).

2000 Mathematics Subject Classification: Primary 37C25; Secondary 55M20.

Key words and phrases: periodic points, minimal period, cohomology algebra, Lefschetz number, transversal map.

Research supported by KBN grant No. 2 PO3A 03315. 
Consequently, these conditions, and other forced by the form of the induced map $f^{*}$ or by the structure of $H^{*}(X ; \mathbb{Q})$, may be useful in finding $m$-periodic points.

Another way of gathering additional information about the local fixed point index is possible by putting some analytical or geometrical conditions on $f$. A typical example is the result of Shub and Sullivan [SS] which states that the sequence of local indices of a $C^{1}$ map $f$ at an isolated fixed point $x_{0}$ is bounded provided it is well defined. From this fact it follows that a $C^{1}$ map $f$ of a compact manifold has infinitely many periodic points if the sequence $\left\{L\left(f^{m}\right)\right\}_{m=1}^{\infty}$ is unbounded. This theorem was improved by Chow, Mallet-Paret and Yorke [CMPY] and also by Babenko and Bogatyı [BB], who proved that the sequence of fixed point indices is an integral linear combination of elementary periodic sequences with periods determined by the spectrum of the derivative $D f\left(x_{0}\right)$ of $f$ at $x_{0}$.

The comparison of the so-called $k$-adic expansion of $\left\{I\left(f^{m}, X\right)\right\}_{m=1}^{\infty}$ with the same expansion of $\left\{L\left(f^{m}\right)\right\}_{m=1}^{\infty}$ gives the existence of minimal periods for transversal maps provided the cohomology ring of $X$ has a special form (e.g. $X$ is a sphere or a projective space) $[\mathrm{M}]$.

Jiang and Llibre have recently discussed the arithmetic of the sequence $\left\{\operatorname{det}\left(I-A^{m}\right)\right\}_{m=1}^{\infty}$, where $A$ is an integral square matrix, to apply it to the study of the minimal periods of torus maps [JL]. Using a deep fact on algebraic numbers they showed that for every $m>m_{0}(X)$ for which $L\left(f^{m}\right) \neq 0, m$ is an algebraic period, i.e. $i_{m}(f)=\sum_{k / m} \mu(k) L\left(f^{m / k}\right) \neq 0$. For a torus map this implies that $m$ is a minimal period, since there is equality, up to sign, of the Lefschetz and Nielsen numbers [JL].

On the other hand Haibao $[\mathrm{H}]$ observed that for self-maps of so-called rational exterior spaces we have a formula for the Lefschetz number of the iterated map: $L\left(f^{m}\right)=\operatorname{det}\left(I-A^{m}\right)$, where $A$ is an integral $k \times k$ matrix with $k$ depending on $X$ but independent of $f$.

In this paper we show that the algebraic number theorem of [JL] can be adapted to study minimal periods of self-maps of rational exterior spaces in view of the Haibao theorem. We consider the class of so-called essential maps. For self-maps $f$ of rational exterior spaces the requirement is that $\left\{L\left(f^{n}\right)\right\}_{n=1}^{\infty}$ be unbounded (Prop. 3.13). The main results of this paper are the following.

We show that there exists a constant $m_{X}$ depending only on the space $X$ (more precisely on the dimension of the matrix $A$ ) such that for every essential self-map $f$ of a rational exterior space and all $m>m_{X}$ with $L\left(f^{m}\right) \neq 0$, $m$ is an algebraic period $\left(i_{m}(f) \neq 0\right)$ (Th. 5.1). As a consequence for the class of transversal maps we show that if $m>m_{X}$ then $m$ is a minimal period of $f$ if $m$ is odd, and either $m / 2$ or $m$ is a minimal period of $f$ if $m$ is even (Th. 6.1). This generalizes the results from [M] and [CLN] to the 
class of rational exterior spaces. We also indicate another class of spaces for which this remains true (simple rational Hopf spaces, cf. Def. 4.1).

For $C^{1}$ maps we prove that almost all primes are minimal periods of each essential self-map of a rational exterior compact manifold (Th. 7.3), which is a refinement of a result of Marzantowicz and Przygodzki who noticed the presence of an infinite sequence of primes among the minimal periods of a compact manifold $X$ such that $\operatorname{dim} H_{i}(X ; \mathbb{Q}) \leq 1[\mathrm{MP}]$.

Under the assumption that $X$ is a rational exterior space (or simple rational Hopf space) we give a refined version of the estimate for the number of periodic orbits of a $C^{1}$ self-map of a compact manifold proved by Babenko and Bogatyı̆ [BB] (Th. 7.4).

2. Dold's relations and transversal maps. For the rest of the paper we make the following assumption: if $X$ is a manifold then we only consider self-maps $f$ of $X$ such that all fixed points of $f^{n}$ for every $n$ are isolated and contained in Int $X$.

In this section we recall the relations among elements of the sequence $\left\{I\left(f^{m}, X\right)\right\}_{n=1}^{\infty}$ for self-maps of ENRs, where $I(f)$ denotes the fixed point index of $f$. We also define the class of transversal maps and list their properties connected with the behaviour of $\left\{I\left(f^{m}, X\right)\right\}_{n=1}^{\infty}$.

If $f$ is a self-map of a compact ENR and $I(f)$ is the fixed point index of $f$ in $X$, then there are some important relations between $I\left(f^{m}\right)$ for distinct $m$. For every $m \in \mathbb{N}$ define

$$
i_{m}(f)=\sum_{k \mid m} \mu(k) I\left(f^{m / k}\right),
$$

where $\mu(k)$ denotes the Möbius function (cf. [Ch]).

Then the following congruences (called Dold's relations) hold [D]:

2.1. Proposition. For every $m \in \mathbb{N}$ we have $i_{m}(f) \equiv 0(\bmod m)$.

This formula has a clear interpretation for a self-map $f$ of a discrete countable set $X$. In that case we have $\left|P_{m}(f)\right|=i_{m}(f)$ and the congruence in 2.1 results from the fact that $P_{m}(f)$ consists of $m$-orbits, i.e. the orbits which consist of points with minimal period $m([\mathrm{D}])$.

2.2. Definition (cf. [D], [Mats]). Let $f: X \rightarrow X$ be a $C^{\infty}$ map of an open subset of a manifold $X$. We say that $f \in \Delta$, or that $f$ is a transversal $m a p$, if for any $m \in \mathbb{N}$ and $x \in P^{m}(f)$ we have $1 \notin \sigma\left(D f^{m}(x)\right)$.

Notice that if $f \in \Delta$ and $x \in P_{m}(f)$ then the Hopf formula gives

$$
I\left(f^{m}, x\right)=\operatorname{sign} \operatorname{det}\left(I-D f^{m}(x)\right) \text {. }
$$

We can divide $P_{m}(f)$ into a disjoint union $P_{m}^{\mathrm{E}}(f) \cup P_{m}^{\mathrm{O}}(f)$, depending on whether the index is 1 or -1 . We say that $x \in P_{m}(f)$ is a twisted 
m-periodic point if $I\left(f^{m}, x\right)=-I\left(f^{2 m}, x\right)$, and is nontwisted otherwise. In this way we can split $P_{m}^{\mathrm{E}}(f)$ and $P_{m}^{\mathrm{O}}(f)$ as $P_{m}^{\mathrm{E}}(f)=P_{m}^{\mathrm{EE}}(f) \cup P_{m}^{\mathrm{EO}}(f)$, $P_{m}^{\mathrm{O}}(f)=P_{m}^{\mathrm{OE}}(f) \cup P_{m}^{\mathrm{OO}}(f)$, where

$$
\begin{aligned}
& P_{m}^{\mathrm{EE}}(f)=\left\{x \in \operatorname{Per}\left(f^{m}\right): \sigma_{+}(x), \sigma_{-}(x) \text { are even }\right\}, \\
& P_{m}^{\mathrm{EO}}(f)=\left\{x \in \operatorname{Per}\left(f^{m}\right): \sigma_{+}(x) \text { is even, } \sigma_{-}(x) \text { is odd }\right\}, \\
& P_{m}^{\mathrm{OE}}(f)=\left\{x \in \operatorname{Per}\left(f^{m}\right): \sigma_{+}(x) \text { is odd, } \sigma_{-}(x) \text { is even }\right\}, \\
& P_{m}^{\mathrm{OO}}(f)=\left\{x \in \operatorname{Per}\left(f^{m}\right): \sigma_{+}(x), \sigma_{-}(x) \text { are odd }\right\},
\end{aligned}
$$

and $\sigma_{+}(x)$ (resp. $\left.\sigma_{-}(x)\right)$ denotes the number of real eigenvalues of $D\left(f^{m}(x)\right)$ greater than one (smaller than -1 respectively) counted with multiplicity. The set $P_{m}^{\mathrm{tw}}(f)=P_{m}^{\mathrm{EO}}(f) \cup P_{m}^{\mathrm{OO}}(f)$ denotes the set of twisted points.

For the class of transversal maps we have the following Dold equalities (cf. $[\mathrm{D}]$ ).

2.3. Proposition. If $f$ is transversal, then

$$
\begin{aligned}
\left(D_{\text {odd }}\right) & i_{m}(f)=\sum_{x \in P_{m}(f)} I\left(f^{m}, x\right) \quad \text { if } m \text { is odd } \\
\left(D_{\text {even }}\right) & i_{m}(f)=\sum_{x \in P_{m}(f)} I\left(f^{m}, x\right)-2 \sum_{x \in P_{m / 2}^{\mathrm{tw}}(f)} I\left(f^{m / 2}, x\right)
\end{aligned}
$$

if $m$ is even,

which can also be written in the form

$$
\begin{aligned}
&\left(D_{\text {odd }}^{\prime}\right) i_{m}(f)=\left|P_{m}^{\mathrm{E}}(f)\right|-\left|P_{m}^{\mathrm{O}}(f)\right| \quad \text { if } m \text { is odd, } \\
&\left(D_{\text {even }}^{\prime}\right) i_{m}(f)=\left|P_{m}^{\mathrm{E}}(f)\right|-\left|P_{m}^{\mathrm{O}}(f)\right|-2\left(\left|P_{m / 2}^{\mathrm{EO}}(f)\right|-\left|P_{m / 2}^{\mathrm{OO}}(f)\right|\right) \\
& \text { if } m \text { is even. }
\end{aligned}
$$

2.4. Definition. A natural number $m$ is called an algebraic period of a self-map $f$ if $i_{m}(f) \neq 0$.

2.5. Corollary. Let $f$ be a transversal self-map of a compact manifold $X$ and let $m$ be an algebraic period of $f$. Then $m$ is a minimal period for $m$ odd, and either $m$ or $m / 2$ is a minimal period for $m$ even.

Proof. An immediate consequence of Dold's equalities $\left(D_{\text {odd }}\right)$ and $\left(D_{\text {even }}\right)$.

Let $\operatorname{Or}(f, m)$ denote the number of $m$-orbits of $f$.

2.6. Proposition. Let $f$ be a transversal self-map of a compact manifold $X$. Then for every odd $m$,

$$
\operatorname{Or}(f, m) \equiv i_{m}(f)(\bmod 2) .
$$


Proof. By $\left(D_{\text {odd }}^{\prime}\right)$ we have

$$
\operatorname{Or}(f, m)=\left|P_{m}(f)\right| / m=\left(\left|P_{m}^{\mathrm{E}}\right|+\left|P_{m}^{\mathrm{O}}\right|\right) / m=2\left|P_{m}^{\mathrm{O}}\right| / m+i_{m}(f) / m,
$$

which gives the assertion.

3. Lefschetz numbers for maps on rational exterior spaces. We now briefly sketch the main result of Haibao's paper $[\mathrm{H}]$ and prove some facts about the growth of the sequence $\left\{L\left(f^{m}\right)\right\}_{m=1}^{\infty}$ for a self-map of a rational exterior space.

For a given space $X$ and an integer $r \geq 0$ let $H^{r}(X ; \mathbb{Q})$ be the $r$ th singular cohomology space with rational coefficients. Let $H^{*}(X ; \mathbb{Q})=\bigoplus_{r=0}^{s} H^{r}(X ; \mathbb{Q})$ be the cohomology algebra with multiplication given by the cup product. An element $x \in H^{r}(X ; \mathbb{Q})$ is decomposable if there are pairs $\left(x_{i}, y_{i}\right) \in$ $H^{p_{i}}(X ; \mathbb{Q}) \times H^{q_{i}}(X ; \mathbb{Q})$ with $p_{i}, q_{i}>0, p_{i}+q_{i}=r>0$ so that $x=\sum x_{i} \cup y_{i}$. Let $A^{r}(X)=H^{r}(X) / D^{r}(X)$, where $D^{r}$ is the linear subspace of all decomposable elements. For a continuous map $f: X \rightarrow X$ let $f^{*}$ be the induced homomorphism on cohomology and $A(f)$ the induced homomorphism on $A(X)=\bigoplus_{r=0}^{s} A^{r}(X)$.

3.1. Definition. Let $f$ be a self-map of a space $X$ and let $I: A(X) \rightarrow$ $A(X)$ be the identity morphism. The polynomial

$$
A_{f}(t)=\operatorname{det}(t I-A(f))=\prod_{r \geq 1} \operatorname{det}\left(t I-A^{r}(f)\right)
$$

will be called the characteristic polynomial of $f$. The zeros of this polynomial: $\lambda_{1}(f), \ldots, \lambda_{k}(f), k=\operatorname{rank} X$, where $\operatorname{rank} X$ is the dimension of $A(X)$ over $\mathbb{Q}$, will be called the quotient eigenvalues of $f$.

3.2. TheORem $([\mathrm{H}])$. If $f$ is a self-map of a space $X$, then $A_{f}(t) \in \mathbb{Z}[t]$. Moreover, if $\operatorname{dim} A^{r}(X)$ is either 1 or 0 for all $r \geq 1$, then the quotient eigenvalues $\lambda_{1}(f), \ldots, \lambda_{k}(f)$ are all integers and $A_{f}(t)=\prod_{i=1}^{k}\left(t-\lambda_{i}(f)\right)$.

Now we introduce the class of rational exterior spaces.

3.3. Definition. A connected topological space $X$ is called rational exterior if there are some homogeneous elements $x_{i} \in H^{\text {odd }}(X ; \mathbb{Q}), i=1, \ldots, k$, such that the inclusions $x_{i} \hookrightarrow H^{*}(X ; \mathbb{Q})$ give rise to a ring isomorphism $\Lambda_{\mathbb{Q}}\left(x_{1}, \ldots, x_{k}\right)=H^{*}(X ; \mathbb{Q})$. Additionally if the set $\left\{x_{i}\right\}_{i=1}^{k}$ can be ordered so that $\operatorname{dim} x_{1}<\ldots<\operatorname{dim} x_{k}$, we call $X$ a simple rational exterior space.

The rational exterior spaces are a wide class of spaces that encompass: finite $H$-spaces, including all finite-dimensional Lie groups and some real Stiefel manifolds, and spaces that admit a filtration

$$
\left.X=X_{0} \stackrel{p_{0}}{\longrightarrow} X_{1} \stackrel{p_{1}}{\longrightarrow} \ldots \stackrel{p_{k-1}}{\longrightarrow} X_{k} \stackrel{p_{k}}{\longrightarrow} X_{k+1}=\text { \{point }\right\}
$$

where $p_{i}$ is the projection of an odd-dimensional sphere bundle $[\mathrm{H}]$. 
The Lefschetz number for self-maps of a rational exterior space can be expressed in terms of quotient eigenvalues.

3.4. TheOREM $([\mathrm{H}])$. Let $f$ be a self-map of a rational exterior space and $A_{f}(t)$ be the characteristic polynomial of $f$. Then $L(f)=A_{f}(1)$.

We can repeat the construction of $A(f)$, given at the beginning of this section, for cohomology with integer coefficients. Consider the cohomology group $H^{r}(X ; \mathbb{Z})$ and its subgroup $B^{r}(X ; \mathbb{Q})$ generated by all $r$-dimensional decomposable elements. Define $\widetilde{A}^{r}(X)=H^{r}(X) / B^{r}(X), r>0$. Let $\widetilde{A}(f)$ be the homomorphism induced by $f$ on $\widetilde{A}(X)=\bigoplus_{r=0}^{s} \widetilde{A}^{r}(X)$, and $\widetilde{A}_{f}(t)$ be the characteristic polynomial of $f$ on $\widetilde{A}(X)$. Then (cf. [H], Lemmas 4.2 and 4.3) $\widetilde{A}^{r}(X)$ is a free $\mathbb{Z}$-module, $\operatorname{rank}_{\mathbb{Z}} \widetilde{A}^{r}(X)=\operatorname{dim}_{\mathbb{Q}} A^{r}(X)$ and

$$
A_{f}(t)=\widetilde{A}_{f}(t) \text {. }
$$

As a consequence we obtain:

3.5. Theorem $([\mathrm{H}])$. Let $f$ be a self-map of a rational exterior space, and let $\lambda_{1}, \ldots, \lambda_{k}$ be the quotient eigenvalues of $f$. Let $A$ denote the integral matrix of $\widetilde{A}(f)$. Then $L\left(f^{n}\right)=\operatorname{det}\left(I-A^{n}\right)=\prod_{i=1}^{k}\left(1-\lambda_{i}^{n}\right)$.

The sequence $\left\{\operatorname{det}\left(I-A^{m}\right)\right\}_{m=1}^{\infty}=\left\{L\left(f^{m}\right)\right\}_{m=1}^{\infty}$, where $A$ is an integral square matrix, has a nice arithmetic structure, which was observed by Jiang and Llibre [JL] for self-maps of tori. The algebraic framework of their paper was developed in order to obtain a complete description of the minimal set of homotopy periods of a torus map $f: T^{r} \rightarrow T^{r}$ defined as $\operatorname{MPer}(f)=$ $\bigcap_{g \simeq f} \operatorname{Per}(g)$, where $g$ is homotopic to $f$. The topological part of their work bases on the fact that for self-maps of tori we have $\left|L\left(f^{m}\right)\right|=N\left(f^{m}\right) \geq 0$, where $N\left(f^{m}\right)$ is the Nielsen number of $f^{m}$, which is the lower bound for the number of fixed points of $f^{m}$.

Although rational exterior spaces do not have such a nice property, the algebraic structure of $\left\{L\left(f^{n}\right)\right\}_{n=1}^{\infty}$ is the same as in the case considered by Jiang and Llibre. This makes it possible to use their results to find minimal periods of self-maps of rational exterior spaces.

For a square matrix $G \in M_{r \times r}(\mathbb{Z})$, we define $F_{G}(m):=\left|\operatorname{det}\left(I-G^{m}\right)\right|$ and $T_{G}:=\left\{m \in \mathbb{N}: F_{G}(m) \neq 0\right\}$.

Let $\varrho$ be the spectral radius of $G$, i.e. the maximal modulus of eigenvalues of $G$.

3.6. TheOREM ([JL]). There exists $m_{0}(r)$ such that for every $G \in M_{r \times r}(\mathbb{Z})$ with $\varrho>1$ and all $m, n \in T_{G}$ with $n \mid m, m>m_{0}(r)$ we have

$$
F_{G}(m) / F_{G}(n)>1 \text {. }
$$

3.7. Remark. The number $m_{0}(r)$ is effectively computable. 
As a matter of fact Theorem 3.6 in this formulation easily follows from the classical Schinzel theorem on primitive divisors (cf. [Sch], [JM]). However, Jiang and Llibre gave a proof which was based on some nontrivial inequalities for algebraic numbers.

We have the following modification of Theorem 3.6.

3.8. Lemma. Let $\varepsilon=\varepsilon(m)$ be a fixed sequence of positive numbers such that

$$
\limsup _{n \rightarrow \infty} \varepsilon(m)<1 .
$$

Then there exists a natural number $m(r, \varepsilon)$ such that for every $G \in M_{r \times r}(\mathbb{Z})$ with $\varrho>1$ and all $m, n \in T_{G}$ with $n \mid m$ and $m>m(r, \varepsilon)$ we have

$$
F_{G}(m) / F_{G}(n)>\varrho^{\varepsilon(m) m / 2} .
$$

Pr o of. Assume that $m \geq 5000$, so that $\ln m \geq 8.5$. It is known (cf. [JL]) that

$$
F_{G}(m) / F_{G}(n)>\frac{\varrho^{m / 2}-1}{e^{9 r(41.4+(r / 2) \ln \varrho)(r \ln m)^{2}}} .
$$

Consider the inequality

$$
\frac{\varrho^{m / 2}-1}{e^{9 r(41.4+(r / 2) \ln \varrho)(r \ln m)^{2}}}>\varrho^{\varepsilon(m) m / 2} .
$$

It is obvious that for every fixed $\varrho>1$ it is satisfied for sufficiently large $m$. We want to find $m(r, \varepsilon)$ such that it is valid for all $m>m(r, \varepsilon)$ independently of the choice of $\varrho>1$.

Following the arguments of [JL] consider two cases. If $\varrho \geq e^{82.8 / r}$ then

$$
41.4+(r / 2) \ln \varrho \leq r \ln \varrho,
$$

so that $(* *)$ holds provided

$$
(* * *) \quad \varrho^{m / 2}>\varrho^{\varepsilon(m) m / 2+9 r^{4}(\ln m)^{2}}+1 .
$$

As $\varrho>e^{82.8 / r}$ we have $\varrho>1+82.8 / r$ and $(* * *)$ is valid if

$$
\frac{m}{2}(1-\varepsilon(m))>9 r^{4}(\ln m)^{2}+1 .
$$

Let $m_{1}(r, \varepsilon)$ be such that the last inequality is satisfied for all $m>m_{1}(r, \varepsilon)$. Then $(* * *)$ and consequently $(* *)$ are satisfied for all $m>m_{1}(r, \varepsilon)$.

The remaining case $\varrho<e^{82.8 / r}$ leads to a finite number of possible characteristic polynomials $\chi_{G}(\lambda)$ of $G$ as the coefficients of $\chi_{A}(\lambda)$ are elementary symmetric polynomials in the eigenvalues and so can be estimated by $\varrho$. We then choose the smallest $\varrho$ of the corresponding characteristic polynomials, say $\varrho_{0}$, and let $m_{2}(r, \varepsilon)$ be such that (**) is satisfied for $\varrho_{0}$ and $m>m_{2}(r, \varepsilon)$. Then $(*)$ holds for all $m>m(r, \varepsilon)=\max \left(5000, m_{1}(r, \varepsilon), m_{2}(r, \varepsilon)\right)$. 
3.9. Definition. A map $f$ will be called essential provided:

(a) 1 is not its quotient eigenvalue,

(b) at least one quotient eigenvalue is neither zero nor a primitive root of unity.

Kronecker Theorem (cf. [N]). Let $\varrho$ be the spectral radius of $G \in$ $M_{r \times r}(\mathbb{Z})$. If $\varrho \leq 1$, then all non-zero eigenvalues of $G$ are roots of unity.

3.10. TheOREM. Let $\varepsilon(m)$ be a sequence of positive numbers such that

$$
\limsup _{m \rightarrow \infty} \varepsilon(m)<1 .
$$

Then there exists a natural number $m(k, \varepsilon)$ such that for every essential self-map $f$ of a rational exterior space of rank $k$ and all $m, n \in T_{A}$ with $n \mid m$ and $m>m(k, \varepsilon)$ we have

$$
\left|L\left(f^{m}\right)\right| /\left|L\left(f^{n}\right)\right|>\varrho^{\varepsilon(m) m / 2},
$$

where $\varrho$ is the spectral radius of the matrix $A \in M_{k \times k}(\mathbb{Z})$ of $\widetilde{A}(f)$.

Proof. Since $f$ is essential, by Definition 3.9(b) and the Kronecker Theorem the spectral radius $\varrho$ of $A$ satisfies $\varrho>1$. We have $F_{A}(m)=$ $\left|\operatorname{det}\left(I-A^{m}\right)\right|$, so due to Theorem $3.5, F_{A}(m)=\left|L\left(f^{m}\right)\right|$, and finally by Lemma 3.8 we complete the proof.

3.11. REMARK. The structure of the sequence $\left\{L\left(f^{n}\right)\right\}_{n=1}^{\infty}$ for rational exterior spaces has a description in terms of cyclotomic polynomials. Let $\psi_{d}(x)$ be the $d$ th cyclotomic polynomial. Then by the identity $x^{m}-1=$ $\prod_{d \mid m} \psi_{d}(x)$ we see that

$$
\left|L\left(f^{m}\right)\right|=\left|\operatorname{det}\left(1-A^{m}\right)\right|=\prod_{d \mid m}\left|\operatorname{det} \psi_{d}(A)\right|=\prod_{d \mid m} \Psi_{d},
$$

where $\Psi_{d}=\left|\operatorname{det} \psi_{d}(A)\right|$.

The coefficients of $\psi_{d}$ are integers and $A$ is an integer matrix as well, so $\Psi_{d}$ is an integer for every $d$. As a consequence we obtain:

3.12. ThEOREM. Let $f$ be a self-map of a rational exterior space and $n \mid m$, $n \in T_{A}$. Then $L\left(f^{n}\right) \mid L\left(f^{m}\right)$.

Theorem 3.10 and Remark 3.11 make it possible to give a characterization of essential maps on rational exterior spaces.

3.13. Proposition. A self-map $f$ of a rational exterior space is essential iff $\left\{L\left(f^{m}\right)\right\}_{m=1}^{\infty}$ is unbounded.

Pro of. If $f$ is essential then $\left\{L\left(f^{m}\right)\right\}_{m=1}^{\infty}$ is unbounded by Lemma 3.8. If $f$ is not essential then all its non-zero quotient eigenvalues $\lambda_{1}, \ldots, \lambda_{k}$ are roots of unity, each being a root of some cyclotomic polynomial $\psi_{n_{i}}$ of degree 
$d_{i} \leq k=\operatorname{rank} X$. Let $C=\operatorname{lcm}\left\{d_{i}: i=1, \ldots, k\right\}$. Obviously $\lambda_{i}^{C}=1$ and so we have

$$
L\left(f^{m+C}\right)=\prod_{i=1}^{k}\left(1-\lambda_{i}^{m+C}\right)=\prod_{i=1}^{k}\left(1-\lambda_{i}^{m}\right)=L\left(f^{m}\right),
$$

thus $\left\{L\left(f^{m}\right)\right\}_{m=1}^{\infty}$ is periodic and consequently bounded (cf. [JL]).

3.14. REMARK. For rational exterior spaces there are some restrictions on the integers which may appear in the sequence $\left\{L\left(f^{m}\right)\right\}_{m=1}^{\infty}$, besides Dold's relations. Namely there is $M$ such that for all $m>M$ the divisors of $L\left(f^{m}\right)$ must be primitive. This means that for every $m>M$ there is a prime number $p$ such that $p \mid L\left(f^{m}\right)$ but $p \nmid L\left(f^{n}\right)$ for $n<m$. The number $M$ is usually very large (cf. [Sch]).

4. A formula for simple rational Hopf spaces. Theorem 3.5 does not cover the cases when the generators of $H^{*}(X ; \mathbb{Q})$ are in even-dimensional cohomology, so it does not embrace the case of $S^{2 n}$ and other similar spaces. However, it is possible to extend Haibao's method to find a formula for the Lefschetz number for a wider class of spaces.

4.1. Definition. A connected topological space $X$ is called a simple rational Hopf space if there are homogeneous elements $x_{i} \in H^{\text {odd }}(X ; \mathbb{Q})$, $y_{j} \in H^{\text {even }}(X ; \mathbb{Q}), i=1, \ldots, k, j=1, \ldots, l$, such that the inclusions $x_{i} \hookrightarrow H^{*}(X ; \mathbb{Q}), y_{j} \hookrightarrow H^{*}(X ; \mathbb{Q})$ give rise to an algebra isomorphism $H_{\mathbb{Q}}\left(x_{1}, \ldots, x_{k}, y_{1}, \ldots, y_{l}\right)=H^{*}(X ; \mathbb{Q})$, where $H_{\mathbb{Q}}$ is the free skew-commutative graded algebra with the additional relations $y_{j}^{d_{j}+1}=0$, and the set $\left\{z_{i}\right\}_{i=1}^{k+l}=\left\{x_{i}\right\}_{i=1}^{k} \cup\left\{y_{j}\right\}_{i=1}^{l}$ can be ordered so that $\operatorname{dim} z_{1}<\ldots<\operatorname{dim} z_{k+l}$.

Let $1 \in H^{0}(X ; \mathbb{Q})$ be the unit cocycle. Then $\left\{x_{i}\right\}_{i=1}^{k} \cup\left\{y_{j}\right\}_{i=1}^{l}$ is a vector space basis for $A(X)$ and $B=\left\{1, x_{i_{1}} \cup \ldots \cup x_{i_{n}} \cup y_{j_{1}}^{p_{j_{1}}} \cup \ldots \cup y_{j_{m}}^{p_{j_{m}}}: 1 \leq\right.$ $\left.i_{1}<\ldots<i_{n} \leq k, 1 \leq j_{1}<\ldots<j_{m} \leq l, 1 \leq p_{j_{t}} \leq d_{j_{t}}\right\}$ is a vector space basis for $H^{*}(X ; \mathbb{Q})$. We will use the following notation: $D=k+\sum_{j=1}^{l} d_{j}$, $\operatorname{dim} \lambda_{i}=p$ if $A(f)\left(z_{i}\right)=\lambda_{i} z_{i}$ and $z_{i} \in A^{p}(X)$. The following theorem is a consequence of Haibao's computation (cf. $[\mathrm{H}]$ ).

4.2. Theorem. If $f$ is a self-map of a simple rational Hopf space $X$ with the non-zero quotient eigenvalues $\lambda_{1}, \ldots, \lambda_{k}$ having odd-dimensional eigenvectors and $\lambda_{k+1}, \ldots, \lambda_{k+l}$ having even-dimensional eigenvectors, then

$$
\begin{gathered}
L\left(f^{m}\right)=1+\ldots+(-1)^{\sum_{r=1}^{s} \operatorname{dim} \lambda_{g_{r}}}\left(\lambda_{g_{1}} \ldots \lambda_{g_{s}}\right)^{m}+\ldots \\
\ldots+(-1)^{D}\left(\lambda_{1} \ldots \lambda_{k} \lambda_{k+1}^{d_{k+1}} \ldots \lambda_{k+l}^{d_{k+l}}\right)^{m}, \\
\ldots .
\end{gathered}
$$

where the sum extends over all $1 \leq g_{1}, \ldots, g_{s} \leq k+l$ such that if $g_{t_{1}}=$ $\ldots=g_{t_{w}}$ then $\operatorname{dim} \lambda_{t_{j}}$ is even and $\bar{d}_{t_{w}} \leq w$. 
EXAmples. (A) If $X=S^{2 p}$ then $L\left(f^{m}\right)=1+d^{m}$, where $d=\operatorname{deg} f$.

(B) Consider $X=\mathbb{C P}^{D}$. We have

$$
H^{n}\left(\mathbb{C P}^{D} ; \mathbb{Q}\right)= \begin{cases}\mathbb{Q} & \text { if } n=0,2,4, \ldots, 2 D, \\ 0 & \text { otherwise }\end{cases}
$$

$H^{*}\left(\mathbb{C P}^{D} ; \mathbb{Q}\right)=\operatorname{span}\left\{1, y, y^{2}, \ldots, y^{D}\right\}$ where $0 \neq y \in H^{2}\left(\mathbb{C P}^{D} ; \mathbb{Q}\right)$. If $d=$ $\operatorname{deg} f$, then

$$
L\left(f^{m}\right)=1+d^{m}+d^{2 m}+\ldots+d^{D m} .
$$

(C) Let $X=S^{q} \times S^{q}$, where $q$ is even. Then

$$
H^{n}(X ; \mathbb{Q})= \begin{cases}\mathbb{Q} & \text { if } n=0,2 q, \\ \mathbb{Q} \times \mathbb{Q} & \text { if } n=q, \\ 0 & \text { otherwise, }\end{cases}
$$

and

$$
L\left(f^{m}\right)=1+\lambda_{1}^{m}+\lambda_{2}^{m}+\left(\lambda_{1} \lambda_{2}\right)^{m}
$$

where $\lambda_{1}, \lambda_{2}$ are the eigenvalues of $f^{*}$ on $H^{2 q}(X ; \mathbb{Q})$.

4.3. Definition. If $f$ is a self-map of a simple rational Hopf space which is not a rational exterior space then we will call $f$ essential provided:

(a) 1 and -1 are not its quotient eigenvalues,

(b) at least one of its quotient eigenvalues is different from zero.

5. Algebraic periods. The existence of algebraic periods is an important property of self-maps on rational exterior spaces and simple rational Hopf spaces. For the rest of the paper let $A$ denote the matrix of $\widetilde{A}(f)$. Let $T_{A}=\left\{m \in \mathbb{N}: \operatorname{det}\left(I-A^{m}\right) \neq 0\right\}$.

5.1. THEOREM. Let $X$ be a rational exterior space (or a simple rational Hopf space) of rank $k$. Then there exists a number $m_{X}$ which depends only on the space $X$ such that for every essential self-map $f$ of $X$ each $m \in T_{A}$ with $m>m_{X}$ is an algebraic period of $f$.

Proof. Let $\left|L\left(f^{s}\right)\right|=\max \left\{\left|L\left(f^{m / l}\right)\right|: l \mid m, l \neq m\right\}$. We have

$$
\begin{aligned}
\left|i_{m}(f)\right| & =\left|\sum_{l \mid m} \mu(m / l) L\left(f^{l}\right)\right| \geq\left|L\left(f^{m}\right)\right|-\left|\sum_{l \mid m, l \neq m} \mu(m / l) L\left(f^{l}\right)\right| \\
& \geq\left|L\left(f^{m}\right)\right|-2 \sqrt{m}\left|L\left(f^{s}\right)\right| .
\end{aligned}
$$

The last inequality results from the fact that the number of divisors of $m$ is not greater than $2 \sqrt{m}$ (cf. $[\mathrm{Ch}]$ ).

If $X$ is a rational exterior space, then Theorem 3.10 with $\varepsilon(m)=$ $(2 / m) \log _{\varrho}(2 \sqrt{m})$ yields

$$
\left|L\left(f^{m}\right)\right|>\varrho^{\varepsilon(m) m / 2}\left|L\left(f^{s}\right)\right|=2 \sqrt{m}\left|L\left(f^{s}\right)\right|
$$


for $m>m_{X}=m(k, \varepsilon)$, so that $\left|i_{m}(f)\right|>0$ for $m>m_{X}$. This completes the proof for rational exterior spaces.

If $X$ is a simple rational Hopf space then all quotient eigenvalues are integers. Let $\lambda_{1}, \ldots, \lambda_{D}$ be all quotient eigenvalues of $f$ (assume that they are non-zero but not necessarily different), where $D$ is as in Theorem 4.2, and $\lambda_{1}=\min \lambda_{i}$. By Theorem 4.2 we estimate $L\left(f^{m}\right)$ in the following way:

$$
\left|L\left(f^{m}\right)\right| \geq\left|\lambda_{1} \ldots \lambda_{D}\right|^{m}-2^{D}\left|\lambda_{2} \ldots \lambda_{D}\right|^{m} \geq\left(\left|\lambda_{1}\right|^{m}-2^{D}\right)\left|\lambda_{2} \ldots \lambda_{D}\right|^{m} .
$$

Let now $\left|L\left(f^{s}\right)\right|=\max \left\{\left|L\left(f^{l}\right)\right|: l \mid m, l \neq m\right\}, m=s q$. Then for $m>D$,

$$
\begin{aligned}
\left|i_{m}(f)\right| & \geq\left(\left|\lambda_{1}\right|^{m}-2^{D}\right)\left|\lambda_{2} \ldots \lambda_{D}\right|^{m}-2 \sqrt{m} 2^{D}\left|\lambda_{1} \ldots \lambda_{D}\right|^{s} \\
& \geq\left|\lambda_{2} \ldots \lambda_{D}\right|^{s}\left[\left(\left|\lambda_{1}\right|^{m}-2^{D}\right)\left|\lambda_{2} \ldots \lambda_{D}\right|^{q}-2^{D+1} \sqrt{m} \lambda_{1}^{s}\right] .
\end{aligned}
$$

Obviously there exists $m_{X}$ such that $\left|i_{m}(f)\right| \neq 0$ for all $m>m_{X}$, which completes the proof.

5.2. Remark. Even if $m \notin T_{A}, m$ could be an algebraic period. For example, if $\lambda_{1}, \ldots, \lambda_{r}$ are quotient eigenvalues of an essential self-map of a rational exterior space and each $\lambda_{i}$ is a root of unity of degree $m_{i}(i=$ $1, \ldots, r)$, and all $m_{i}$ are primes, then the number $m=q m_{1} \ldots m_{r}$, where $q \in T_{A}$ and $q>m_{X}$, is an algebraic period.

6. The existence of periodic points for transversal maps. We are now in a position to apply the results of the previous sections to find minimal periods for transversal maps.

6.1. TheOREM. Let $X$ be a rational exterior compact manifold (or a simple rational Hopf space) of rank $r$. Then there exists a number $m_{X}$ which depends only on $X$ such that for every transversal essential self-map $f$ of $X$ and for all $m>m_{X}, m \in T_{A}$ we have: $m$ is odd implies $m \in \operatorname{Per}(f) ; m$ is even implies $m \in \operatorname{Per}(f)$ or $m / 2 \in \operatorname{Per}(f)$.

Pr o o f. According to Corollary 2.5 it suffices to show that $m$ is an algebraic period, and this follows from Theorem 5.1.

The number of periodic points for transversal self-maps of rational exterior spaces grows at exponential rate. Let $\mathrm{Or}_{\mathrm{tw}}(m)$ denote the number of $m$-orbits which consist only of twisted $m$-periodic points.

6.2. Theorem. Let $X$ be a rational exterior compact manifold of rank $r$ and $f: X \rightarrow X$ be an essential transversal map. Set $\operatorname{Or}(m)=\operatorname{Or}(f, m)$. Then for every fixed $0<\alpha<1$ there exists a number $m(r, \alpha)$ such that for all $m>m(r, \alpha)$,

$$
\operatorname{Or}(m) \geq \frac{1}{m}\left[\left(1+\frac{1}{30 r^{2} \ln 6 r}\right)^{\alpha m / 2}-2 \sqrt{m}\right] \text { for } m \text { odd },
$$




$$
\operatorname{Or}(m)+\operatorname{Or}_{\mathrm{tw}}(m / 2) \geq \frac{1}{m}\left[\left(1+\frac{1}{30 r^{2} \ln 6 r}\right)^{\alpha m / 2}-2 \sqrt{m}\right] \text { for } m \text { even. }
$$

Proof. First of all let us quote the following result from the theory of algebraic numbers (cf. $[\mathrm{BM}])$. Let $\widetilde{\varrho}$ be the greatest modulus of conjugate algebraic numbers of degree $n$ over $\mathbb{Q}$. If $\widetilde{\varrho} \neq 0,1$ then

$$
\widetilde{\varrho} \geq 1+\frac{1}{30 n^{2} \ln 6 n} \text {. }
$$

Now we take $\varepsilon(m)=\alpha$, where $0<\alpha<1$ is fixed. Then by Theorem 3.10 we have $\left|L\left(f^{m}\right)\right| /\left|L\left(f^{s}\right)\right|>\varrho^{\alpha m / 2}$ for $m>m(\alpha)$, where $\left|L\left(f^{s}\right)\right|=$ $\max \left\{\left|L\left(f^{l}\right)\right|: l \mid m, l \neq m\right\}$, and consequently, in the same way as in the proof of Theorem 5.1, we obtain

$$
\left|i_{m}(f)\right|>\left(\varrho^{\alpha m / 2}-2 \sqrt{m}\right)\left|L\left(f^{s}\right)\right|
$$

for all $m>m(\alpha)$.

Due to Dold's equalities (2.3), for $m$ odd by $\left(D_{\text {odd }}^{\prime}\right)$ we have

$$
\left|P_{m}(f)\right|=\left|P_{m}^{\mathrm{E}}(f)\right|+\left|P_{m}^{\mathrm{O}}(f)\right| \geq|| P_{m}^{\mathrm{E}}(f)|-| P_{m}^{\mathrm{O}}(f)||=\left|i_{m}(f)\right|,
$$

and for $m$ even by $\left(D_{\text {even }}^{\prime}\right)$,

$$
\begin{aligned}
\left|P_{m}(f)\right|+2\left|P_{m / 2}^{\mathrm{tw}}(f)\right| & =\left|P_{m}^{\mathrm{E}}(f)\right|+\left|P_{m}^{\mathrm{O}}(f)\right|+2\left(\left|P_{m / 2}^{\mathrm{EO}}(f)\right|+\left|P_{m / 2}^{\mathrm{OO}}(f)\right|\right) \\
& \geq|| P_{m}^{\mathrm{E}}(f)|-| P_{m}^{\mathrm{O}}(f)\left|-2\left(\left|P_{m / 2}^{\mathrm{EO}}(f)\right|-\left|P_{m / 2}^{\mathrm{OO}}(f)\right|\right)\right| \\
& =\left|i_{m}(f)\right| .
\end{aligned}
$$

From the equality $\operatorname{Or}(f, m)=\left|P_{m}(f)\right| / m$ applying $(*)$ for $\widetilde{\varrho}=\varrho(r=n)$ and $(* *)$ we finally get the needed estimate for $m>m(\alpha)$ independently of the choice of $f$.

6.3. REMARK. Jiang and Llibre gave an estimate that allows finding $m_{0}$ such that $F_{A}(m) / F_{A}(n)>1$ holds for all $m, n \in T_{A}$ with $m>m_{0}$ and $n \mid m$. For spaces with few non-zero cohomology groups it is however better to examine it explicitly. Considering the case of the three-dimensional torus $T^{3}$ they noticed that according to general theory $m_{0}=10^{5}$, but straightforward calculations show that in fact the set $L$ of $m \in T_{A}$ for which the inequality $F_{A}(m) / F_{A}(n)>1$ may not hold for some $n \in T_{A}$ with $n \mid m$ is $L=\{2,3,4,5,6,8,9,10\}$.

Because Jiang and Llibre base only on the properties of the roots of the characteristic polynomial of a map induced on the cohomology space, we can apply the above result to a space $X$ with $\operatorname{rank} X=3$ in order to obtain some small natural numbers as minimal periods. Let $m_{X}$ be the constant from Theorem 5.1.

6.4. COROLLARY. Let $f$ be an essential transversal self-map of a rational exterior compact manifold $X$ of rank 3 . Let $m<m_{X}, m \in T_{A}, m \notin L, m=$ 
$p^{r} q^{s}$, where $p, q>2$ are different primes such that $\left|L\left(f^{m}\right)\right| /\left|L\left(f^{m /(p q)}\right)\right| \neq 6$. Then $m \in \operatorname{Per}(f)$.

Proof. It is enough to show that $m$ is an algebraic period. We have

$$
\begin{aligned}
\left|i_{m}(f)\right| & =\left|\sum_{l \mid m} \mu(m / l) L\left(f^{l}\right)\right| \\
& =\left|L\left(f^{p^{r-1} q^{s-1}}\right)-L\left(f^{p^{r-1} q^{s}}\right)-L\left(f^{p^{r} q^{s-1}}\right)+L\left(f^{p^{r} q^{s}}\right)\right| .
\end{aligned}
$$

If $l \mid m$ then $L\left(f^{l}\right) \mid L\left(f^{m}\right)$ by Theorem 3.12, thus $L\left(f^{p^{r-1} q^{s}}\right)=$ $a L\left(f^{p^{r-1}} q^{s-1}\right), L\left(f^{p^{r} q^{s-1}}\right)=b L\left(f^{p^{r-1} q^{s-1}}\right), L\left(f^{p^{r} q^{s}}\right)=c L\left(f^{p^{r-1} q^{s-1}}\right)$ and $|a|,|b|,|c|>1$ by Remark 6.3 , because $m \notin L$.

Therefore $\left|i_{m}(f)\right|=\left|L\left(f^{p^{r-1} q^{s-1}}\right)\right||1-a-b+c|$ where $a|c, b| c$ and $a, b$ are proper factors of $c$.

Notice that if $m \in T_{A}$, which is equivalent to $L\left(f^{m}\right) \neq 0$, then by Theorem 3.12, $L\left(f^{s}\right) \neq 0$ for $s \mid m$, thus $L\left(f^{p^{r-1} q^{s-1}}\right) \neq 0$. Let us now consider two cases:

(1) $|a|=|b|=|c| / 2$. Then $\left|i_{m}(f)\right|=\left|L\left(f^{p^{r-1} q^{s-1}}\right)\right|>0$.

(2) $|a| \neq|b|$. Then for $m \notin L$ we obtain

$$
\left|i_{m}(f)\right| \geq\left|L\left(f^{p^{r-1} q^{s-1}}\right)\right|(|c|-|1-a-b|) \geq|c|-(1+|a|+|b|) .
$$

Set $|c|=k_{a}|a|,|c|=k_{b}|b|,|a|>|b|>1$. Notice that $|c|$ must be at least 6 . We want to know when $|c|-(1+|a|+|b|)>0$, or $|a|\left(k_{a}-1\right)>|b|+1$ equivalently. This may not hold only for $k_{a}=2$. In this case $|c| / 2>|c| / 3+1$ (which implies the needed inequality $|c| / 2>|c| / k_{b}+1$ because $k_{b} \geq 3$ ) is satisfied for $|c|>6$. This ends the proof, as the case $|c|=6$ is excluded by assumption.

It is easy to formulate different conditions forcing for $m$ odd that the number of $m$-orbits is even.

6.5. Theorem (cf. $[\mathrm{M}]$ ). Let $f: X \rightarrow X$ be a transversal map, and $X$ be a rational exterior compact manifold. Let $m \in T_{A}$ be an odd number. If either $2 \mid L(f)$ or $2 \nmid L\left(f^{m}\right)$, then

$$
\operatorname{Or}(f, m) \equiv 0(\bmod 2) \text {. }
$$

Pr o of. By Proposition 2.6 we have

$$
\operatorname{Or}(f, m) \equiv i_{m}(f)(\bmod 2) .
$$

On the other hand,

$$
i_{m}(f)=\sum_{l \mid m} \mu(m / l) L\left(f^{l}\right)=\sum_{\tau \subset P(m)}(-1)^{|\tau|} L\left(f^{m: \tau}\right),
$$


where $P(m)$ is the set of all primes which divide $m$, the sum extends over all subsets $\tau$ of $P(m),|\tau|$ stands for the cardinality of $\tau$, and $m: \tau=m / \prod_{p \in \tau} p$ denotes $m$ divided by all $p \in \tau$.

For $s \mid m$ we have $L\left(f^{s}\right) \mid L\left(f^{m}\right)$ by Theorem 3.12; thus if $2 \mid L(f)$, then $2 \mid L\left(f^{s}\right)$ for all $s<m$ and obviously $2 \mid i_{m}(f)$.

If $2 \nmid L\left(f^{m}\right)$, then by Theorem $3.12,2 \nmid L\left(f^{s}\right)$ for all $s \mid m$, so in the sum

$$
i_{m}(f)=\sum_{\tau \subset P(m)}(-1)^{|\tau|} L\left(f^{m: \tau}\right)
$$

there are $2^{P(m)}$ summands. All of them are odd and non-zero because $s \mid m$, $m \in T_{A}$. Thus $2 \mid i_{m}(f)$.

6.6. Theorem. Let $f: X \rightarrow X$ be a transversal self-map of a simple rational Hopf compact manifold. Let $Z(m)=\left\{s \mid m: L\left(f^{s}\right)=0\right\}=\emptyset$. Then for every odd $m$,

$$
\operatorname{Or}(f, m) \equiv 0(\bmod 2)
$$

P r o o f. For integral quotient eigenvalues $\lambda_{1}, \ldots, \lambda_{r}$ of $f$ we have $\lambda_{1} \ldots \lambda_{k}$ $\equiv\left(\lambda_{1} \ldots \lambda_{k}\right)^{m}(\bmod 2)$, and thus

$$
\sum_{1 \leq k_{1} \leq \ldots \leq k_{p} \leq r} \lambda_{k_{1}} \ldots \lambda_{k_{p}} \equiv \sum_{1 \leq k_{1} \leq \ldots \leq k_{p} \leq r}\left(\lambda_{k_{1}} \ldots \lambda_{k_{p}}\right)^{m}(\bmod 2) .
$$

As a consequence, by Theorem 4.2, we obtain

$$
L(f) \equiv L\left(f^{m}\right)(\bmod 2)
$$

for all natural $m$, hence $i_{m}(f)$ is the sum of $2^{P(m)}$ non-zero integers which are either all even or all odd. This gives the statement.

ExAmple. Consider the $D$-dimensional complex projective space $\mathbb{C P}^{D}$. For each odd $m$ and essential transversal $f$ we have $Z(m)=\emptyset$ (cf. Ex. (B) after Theorem 4.2). Thus $\operatorname{Or}(f, m) \equiv 0(\bmod 2)$.

7. Minimal periods for smooth maps. We can find some subsets of $\operatorname{Per}(f)$ in the case of $C^{1}$ self-maps of rational exterior spaces. First of all let us recall a formula for $i_{m}(f)$ for $C^{1}$ self-maps of a compact manifold from $[\mathrm{MP}]$.

Define $O(x) \subset \mathbb{N}$ for $x \in P_{m}(f)$ as $O(x)=\operatorname{Per}\left(D\left(f^{m}(x)\right)\right)$. Recall that $\sigma_{-}$denotes the number of eigenvalues of $D f^{m}(x)$ (counted with multiplicity) smaller than -1 .

7.1. Theorem. Let $f: X \rightarrow X$ be a $C^{1}$ map of a compact manifold $X$. Then for every $l$ there are integers $c_{k}(x)$ such that

$$
i_{l}(f)=\sum_{m k=l} \sum_{x \in P_{m}(f)} c_{k}(x)+\sum_{2 m k=l} \sum_{x \in P_{m}(f)}\left[(-1)^{\sigma_{-}(x) k}-1\right] c_{k}(x)
$$

with the convention that $c_{k}(x)=0$ if $k \notin O(x)$. 
7.2. LEMMA. The structure of the set $O(x)$ is the following (cf. [MP], [CMPY]):

$$
O(x)=\left\{\operatorname{lcm}(K): K \subset \sigma_{(1)}\left(D\left(f^{m}(x)\right)\right)\right\} \cup\{1\}
$$

where $\sigma_{(1)}\left(D\left(f^{m}(x)\right)\right)$ is the set of degrees of primitive roots of unity contained in $\sigma\left(D\left(f^{m}(x)\right)\right)$.

Now we are in a position to formulate the theorem describing the presence of prime minimal periods. Let $\mathcal{P}$ denote the set of prime numbers.

7.3. Theorem. Let $f: X \rightarrow X$ be an essential $C^{1}$ map of a rational exterior compact manifold $X$. Then $\mathcal{P} \backslash \operatorname{Per}(f)$ is finite.

Proof. Substituting $l=p \in \mathcal{P}$ in the formula of Theorem 7.1 we obtain

$$
i_{p}(f)=\sum_{x \in P_{1}(f)} c_{p}(x)+\sum_{x \in P_{p}(f)} c_{1}(x) .
$$

First observe that the set $P_{1}(f)$ is finite since $X$ is compact. Moreover the set $O(x)$ for $x \in P_{1}(f)$ is also finite as a consequence of Lemma 7.2, so by elimination of a finite number of primes from $O(x)$ for each $x \in P_{1}(f)$, for the remaining primes $p$ we obtain

$$
i_{p}(f)=\sum_{x \in P_{p}(f)} c_{1}(x) .
$$

By Theorem 3.10 the left hand side of the above formula is different from 0 for every sufficiently large $p$, which gives the desired conclusion.

Now we present an estimate of the number of periodic points for $C^{1}$ self-maps of rational exterior manifolds.

Let $\bar{O}(x)$ denote the set of algebraic periods at a given point $x$ :

$$
\bar{O}(x)=\left\{s \in \mathbb{N}: i_{s}(f, x)=\sum_{d \mid s} \mu(s / d) I\left(f^{d}, x\right) \neq 0\right\},
$$

and by $G(f, l)$ the set of algebraic periods of $f$ that are no greater than $l$ :

$$
G(f, l)=\left\{s \leq l: i_{s}(f) \neq 0\right\} .
$$

7.4. TheOREM (cf. [BB]). For every rational exterior compact manifold $X$ of dimension $n$ there exists a constant $m_{X}$ such that for all essential $C^{1}$ self-maps $f$ of $X$ we have

$$
O(f, \leq l) \geq \frac{l-m_{X}}{2^{[(n+1) / 2]} \operatorname{dim} H_{*}(M ; \mathbb{Q})},
$$

where $O(f, \leq l)$ is the number of orbits of $f$ with period at most $l$. 
Pro of (cf. also [BB]). If $x$ is an isolated fixed point of a $C^{1}$ self-map of $\mathbb{R}^{n}$ then (cf. $\left.[\mathrm{BB}]\right)$

$$
|\bar{O}(x)| \leq 2^{[(n+1) / 2]} .
$$

Let now $m_{X}$ be the number from Theorem 5.1 such that all $l>m_{X}$, $l \in T_{A}$ are algebraic periods for every $f$. As $f$ is essential, for $l>m_{X}$ at least one number in the interval $\left[l, l+\operatorname{dim} H_{*}(M ; \mathbb{Q})\right)$ belongs to $T_{A}$ and so must be an algebraic period. Consequently, we obtain

$$
|G(f, l)| \geq \frac{l-m_{X}}{\operatorname{dim} H_{*}(M ; \mathbb{Q})} .
$$

On the other hand we have (cf. $[\mathrm{BB}]$ )

$$
i_{s}(f)=\sum_{m \mid s} \sum_{x \in P_{m}(f)} i_{s}(f, x),
$$

Thus

$$
G(f, l) \subset \bigcup_{m \leq l} \bigcup_{x \in P_{m}(f)} \bar{O}(x) .
$$

On the right hand side of the above formula there are no more than $O(f, \leq l)$ components, so by $(*)$ we obtain

$$
|G(f, l)| \leq O(f, \leq l) 2^{[(n+1) / 2]} .
$$

Finally by $(* *)$,

$$
\frac{l-m_{X}}{\operatorname{dim} H_{*}(M ; \mathbb{Q})} \leq O(f, \leq l) 2^{[(n+1) / 2]},
$$

which is the required assertion.

7.5. Remark. Babenko and Bogatyl got the same estimate (cf. [BB]) for a compact manifold, but their constant $m_{X}=m_{f}$ depends on $f$.

7.6. REMARK. For essential self-maps of a compact simple rational Hopf space all natural numbers for $l>m_{X}$ are algebraic periods, thus

$$
O(f, \leq l) \geq \frac{l-m_{X}}{2^{[(n+1) / 2]}},
$$

where $m_{X}$ is the number from Theorem 5.1.

Acknowledgments. I am very grateful to Professor Wacław Marzantowicz for suggesting the problem and many stimulating conversations.

\section{References}

[BB] I. K. Babenko and C. A. Bogaty $\breve{1}$, The behaviour of the index of periodic points under iterations of a mapping, Math. USSR-Izv. 38 (1992), 1-26. 
[BM] P. E. Blanksby and H. L. Montgomery, Algebraic integers near the unit circle, Acta Arith. 18 (1971), 355-369.

[CLN] J. Casasayas, J. Llibre and A. Nunes, Periodic orbits of transversal maps, Math. Proc. Cambridge Philos. Soc. 118 (1995), 161-181.

[Ch] K. Chandrasekharan, Introduction to Analytic Number Theory, Springer, Berlin, 1968.

[CMPY] S. N. Chow, J. Mallet-Paret and J. A. Yorke, A bifurcation invariant: Degenerate orbits treated as a cluster of simple orbits, in: Geometric Dynamics (Rio de Janeiro 1981), Lecture Notes in Math. 1007, Springer, 1983, 109-131.

[D] A. Dold, Fixed point indices of iterated maps, Invent. Math. 74 (1985), 419435.

[H] D. Haibao, The Lefschetz number of iterated maps, Topology Appl. 67 (1995), $71-79$.

[JM] J. Jezierski and W. Marzantowicz, Minimal periods for nilmanifolds, Preprint No 67, Faculty of Mathematics and Informatics UAM, June 1997.

[JL] B. Jiang and J. Llibre, Minimal sets of periods for torus maps, Discrete Contin. Dynam. Systems 4 (1998), 301-320.

[Mats] T. Matsuoka, The number of periodic points of smooth maps, Ergodic Theory Dynam. Systems 9 (1989), 153-163.

[M] W. Marzantowicz, Determination of the periodic points of smooth mappings using Lefschetz numbers and their powers, Russian Math. Izv. 41 (1997), 80-89.

[MP] W. Marzantowicz and P. Przygodzki, Finding periodic points of a map by use of a k-adic expansion, Discrete Contin. Dynam. Systems 5 (1999), 495-514.

[N] W. Narkiewicz, Elementary and Analytic Theory of Algebraic Numbers, PWN, Warszawa, 1974.

[Sch] A. Schinzel, Primitive divisors of the expression $A^{n}-B^{n}$ in algebraic number fields, J. Reine Angew. Math. 268/269 (1974), 27-33.

[SS] M. Shub and P. Sullivan, A remark on the Lefschetz fixed point formula for differentiable maps, Topology 13 (1974), 189-191.

Faculty of Applied Physics and Mathematics

Technical University of Gdańsk

G. Narutowicza 11/12

80-952 Gdańsk, Poland

E-mail: graff@mif.pg.gda.pl 